\title{
The Limitations of Surgical Learning from Watching YouTube VDOs
}

\author{
Kulbhushan Saini $^{1} \cdot$ Arun K. Jain $^{2} \cdot$ Manu Saini $^{2}$ (D) \\ Received: 31 March 2021 / Accepted: 3 May 2021 / Published online: 12 May 2021 \\ (C) Association of Surgeons of India 2021
}

Dear Editor,

"Old is Gold"-Does this well-known idiom fits in today's scenario. The novel surgical teaching and learning pattern continues to evolve over time and it is attributed to the dramatic dissemination of multimedia information that has significantly altered the learning method over the past 20 years [1]. Traditional surgical learning evolves through the stages from 'novice' to 'expert' at a different speed, the surgeon performed the crucial step of the surgery with step by step narration. As the resident becomes more experienced, they are actively guided with required assistance followed by an independent performance of the surgery with passive skilled assistance to ensure patient safety [2]. However, limited viewing system, assistance, and restricted surgical theatre hours demand to have high fidelity virtual simulated learning system and therefore growing thirst among the budding surgeons quenches at easily accessible YouTube platform.

YouTube platform shares thousands of surgical videos in various subspecialties and this is growing by leaps and bounds. Short edited videos on YouTube reinforce and crystalize the information, which would be otherwise difficult to conceptualize in 3D. These YouTube video-based learnings serve as a blessing for the learners, even for the experienced surgeons, where technology seems to have been utilized at its optimal level.

However, this is not the same as practicing and experiencing in the operation theatre. Many surgical videos available on YouTube contain a single view of the surgical field, frequently zoomed in where only the tips of instruments are visible [3]. Understanding procedure steps conceptually is crucial prior to surgery. The ability to master surgical skills requires knowledge of hand positioning as well as dynamic movements of the hands and arms, manipulation or holding of instruments, sutures that are evidently lacking in the YouTube videos [4]. It is worth noting the importance of cognitive skills such as situation awareness, task management, handling complications, and decision-making besides surgical skills that can be taught and pursued only in the real world of operation theatre [5]. YouTube surgical videos provide short-term knowledge and augment the coping ability, not retention ability. Despite these demerits, authors cannot defy the emerging role and support of YouTube videos in the surgical learning, especially in the today's scenario. However, it will be hard to surpass the constructive traditional surgical learning.

\section{Declarations}

Conflict of Interest The authors declare no competing interests.

\section{References}

1. Waljee JF, Chopra V, Saint S (2018) Mentoring millennials. JAMA 319:1547-1548

2. DaRosa DA, Zwischenberger JB, Meyerson SL, George BC, Teitelbaum EN, Soper NJ, Fryer JP (2013) A theory-based model for teaching and assessing residents in the operating room. J Surg Educ 70:24-30

3. Kutz W (2016) Stapedectomy procedure. https://www.youtube.com/ watch?v=FasI4u-h7Mg. Accessed 24 Jan 2020

4. Brown CS, Cunningham CD 3rd, Lee WT, Puscas L (2020) Development of a surgical video atlas for resident education: 3year experience. OTO Open 4:1-5

5. Lotfipour M, Rolius R, Lehman EB, Pantanelli SM, Scott IU (2017) Trends in cataract surgery training curricula. J Cataract Refract Surg 43:49-53

Publisher's Note Springer Nature remains neutral with regard to jurisdictional claims in published maps and institutional affiliations.
Manu Saini

manusaini.1024@gmail.com

1 Department of Anesthesia, PGIMER, Chandigarh, India

2 Department of Ophthalmology, PGIMER, Chandigarh, India 